\title{
An Automatic System Based on Open Source Solutions to Create Virtual Machines of running Linux Systems
}

\author{
Bruno Luigi Martino* \\ CNR-IASI: Istituto di Analisi dei Sistemi ed Informatica \\ Via dei Taurini 1900185 Roma, Italy \\ E-mail: brunolmartino@gmail.com

\section{Memmo Federici} \\ INAF-IASF: Istituto di Astrofisica Spaziale e Fisica Cosmica Roma \\ Via Fosso del Cavaliere 100, 00133 Roma, Italy \\ E-mail: memmo.federicieiaps.inaf.it
}

\begin{abstract}
The goal of this work is to realize a system to allow the automatic cloning of active physical servers (at work and configured) into virtual platforms. This approach is very useful in cases where computer services are provided by server technologically outdated and therefore hard to be upgraded. Moreover, thanks to the intrinsic characteristics of High Reliability of systems based on virtual machines, in case of failure is possible an effective and fast recovery of the working environment ensuring uninterrupted operation.
\end{abstract}

Frontier Research in Astrophysics

26 - 31 May, 2014

Mondello (Palermo), Italy

* Speaker. 


\section{Introduction}

At time of writing of this paper, there are many freeware and commercial systems which promise to carry out the hot cloning of environments between physical and virtualized operating systems Unix like. The tests we carried out have shown their limits clearly demonstrating that none of them can satisfy in full our needs. More in detail, we used the support tools provided with VMware and Xen-server. The way we organize our work, as system administrators, leads to maintain a conservative approach to avoid to follow fashions and trends that force to continuous updates of Operating Systems and application software. This trend generally causes a considerable waste of energy and of time without bringing, in the majority of cases, to significant benefits. The computing cluster AVES, used for the analysis of the scientific data of the INTEGRAL satellite [1], was built in 2008 and the software platform on which it is based is Debian Linux 5. The greatest difficulties related with its use are focused on the reliability level of the cluster master controller (Figure 1); a solution able to ensure the adequate robustness is to use master systems based on virtual machines in Xen hypervisor taking advantage of the characteristics of High Availability outlined by HaVMS [2].

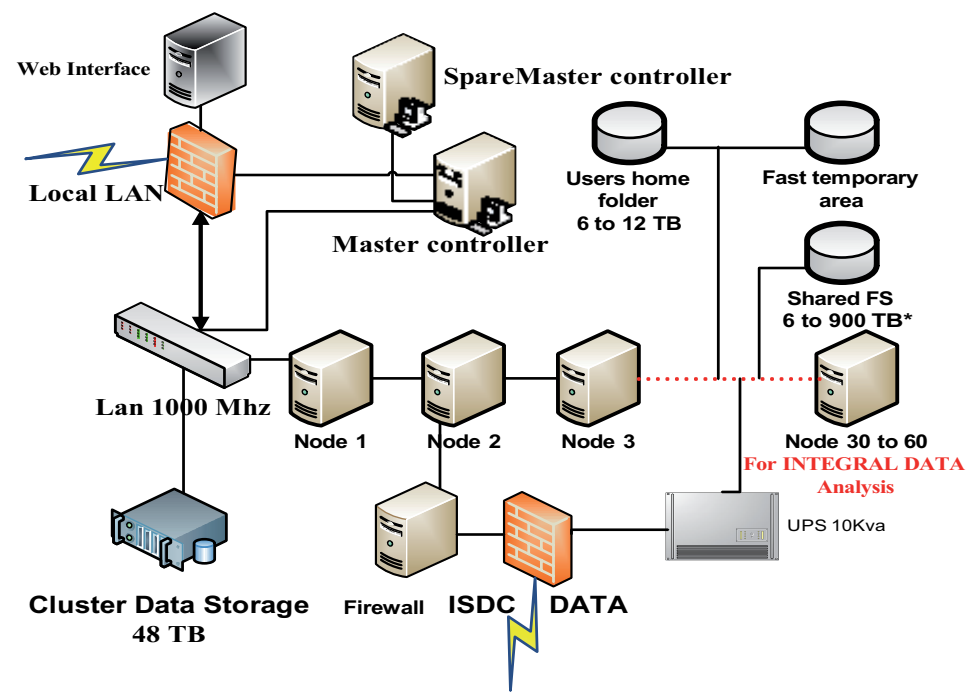

Figure 1: AVES hardware architecture

Unfortunately, the systems used for the hot cloning of XEN (and its competitors) are not able to run on an operating system so dated as that currently in use on AVES [3]. Faced with this barrier the only chance to update the operating system of the cluster seems to be the installation of an operating system most modern, fully supported by the cloning software. Actually this is not a really viable solution as it requires a downtime of the Cluster for unacceptably long time. There is also the chance that the operating system chosen for compatibility with the systems of cloning both in whole or in part, is not compatible with the applications subject of the service provided. To ensure the reliability of the AVES system and increase its longevity we were forced to rely on a custom solution. The methodology we developed is based on a set of scripts made in Bash command language and the use of the XEN-server distribution. 


\section{The context}

The choice of using virtual machines to host the critical elements of a computer system to manage computing services for scientific institutions (using latest generation hardware) enjoys of many benefits.

In fact, technological developments that in computing science that are of particular importance, have lead to significant increases in terms of speed. The transition from one generation to another one of processors (eg pentium pentium 2 Vs vs pentium 4, I3, I5, I7 etc.) and the reduction of cost of memory devices, introduced significant increases in computing performance (unexpected just few years ago), allowing to host on virtualized systems a large number of services and features. It is well known that a virtual machines system has features slightly degraded (a few percent) than comparable systems based on physical platform of the same generation. Nevertheless, the implementation of virtual machines realized with hardware of modern conception of systems based on old or obsolete architectures, allows in any case a good improvement in the overall performance. It's important to emphasize the main advantages of using VM:

- flexibility

- service continuity (HaVMS)

- quick recovery time (snapshot)

\subsection{Flexibility}

A virtual machine image can work under control of an hypervisor on any hardware platform. In our specific case that protects us from the problem of obsolescence of the operating system.

A server hosting virtual machines can accommodate heterogeneous systems having different resources requirements in terms of RAM and CPU, with the only limit of the physical resources made available by it's own hardware; an example of operating environment is shown in Figure 2.

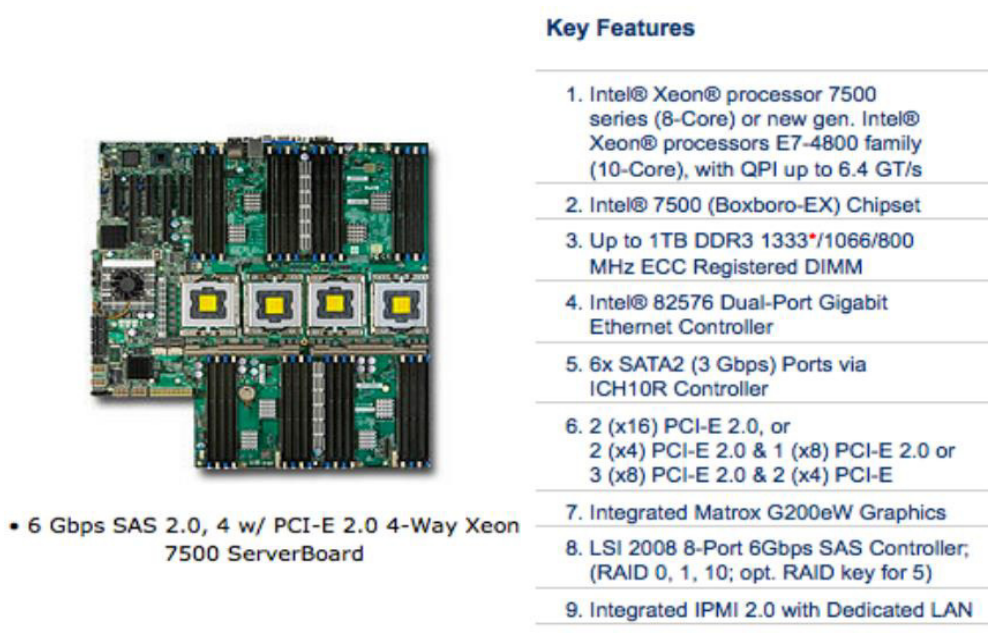

Figure 2: Super Micro motherboard series mp Xeon X9Qxxx 
This motherboard can mount up to 40 cores and 1 TB of RAM; this allows hosting at least 10 high-performance server built on VM performing tasks and duties independent of one another.

\subsection{Service continuity}

Exploiting the characteristics of High Availability provided in the system Xen-server, it's possible to take advantage of a wide range of capabilities to ensure continuity of service, such as the automatic restart of a virtual machine in case of hardware failure or the workload balancing based on availability of resources.

\subsection{Quick recovery time}

By using the technique of snapshots is possible to create restore points which can be used later in case of an unrecoverable machine failure. A special script allows to check the state of health of the VMs; in this way is guaranteed a real continuity of services provided by the individual VM.

\section{Our solution}

As previously mentioned a virtual machine can be created using two different strategies:

- Xen Server conversion tools

- our cloner

The Citrix XenServer Conversion Manager and Citrix XenConvert are tools to convert a workload to XenServer between virtualization platforms and physical machines but unfortunately not every Linux systems (eg. unstable or obsolete Linux OS) can be converted using these tools. CITRIX XEN-SERVER (ver6.x), is provided of an hypervisor to manages theVMs, which allows to define all system parameters such as: RAM, HD, Network, cores and an HA module able, in case of failure, to automatically migrate toward the backup server the running VM, providing uninterrupted service. A VM can be created directly by the cloner or using:

- the Windows tool provided by XEN-SERVER;

- open XEN-MANAGER for Linux and MacOSX;

- Xen VirtualManager (not suitable for paravirtualized VM);

- XEN-TOOLS (able to create paravirtualized VM too)

The main techniques of virtualization are two: full virtualization and paravirtualization. In a fully virtualized machine the physical host (VM server) is used by the VM through a software layerable to share resources with some performance loss; in a paravirtualized system the VMserver avoids the need to emulate a full set of hardware and firmware services; is fast but requires a customized kernel. It is not always possible to use paravirtualized systems. The type of the target machine is the same in both cases and can be considered as some kind of container. That machine must of course be chosen according to the characteristics of the infrastructure to manage (Ram, 
CPU, HD, network).

Our cloner system is composed as follows:

- a client running under an unix like os that can be installed on laptops, VMs or Xen-Server itself;

- a running SSH daemon on source machine;

- a running live linux OS on target machine

It is possible to summarize the cloning process by the following steps:

- SSH connection to source machine;

- compressed backup of source machine disk on target machine;

- SSH connection to the target machine to make bootable the just made copy;

As shown in Figure 3, the cloner is composed by a client that runs a set of scripts able to connect the system to be cloned with the server of the virtual machines based on Xen-server that implements the various phases described previously.

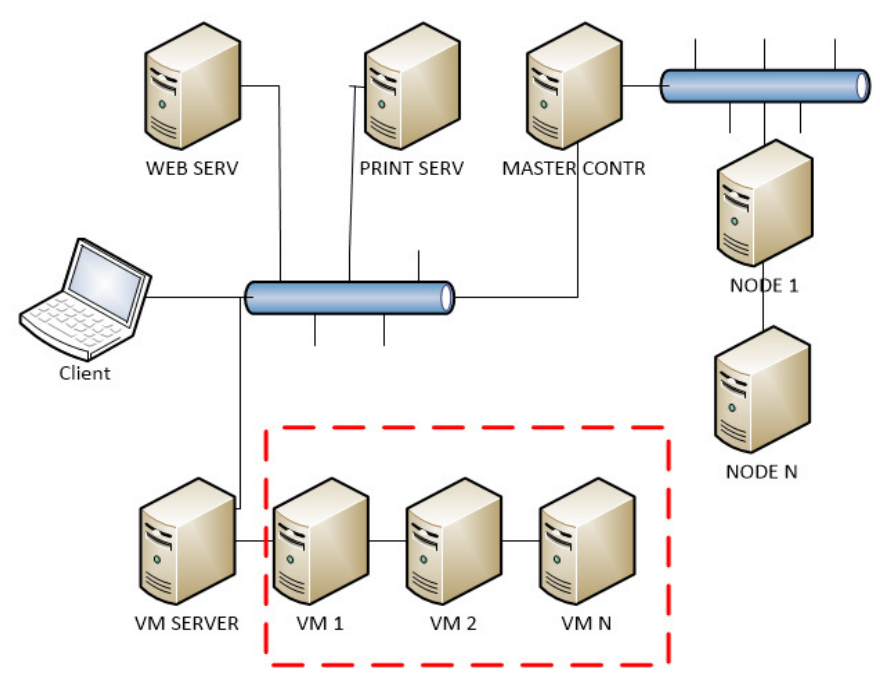

Figure 3: our cloner system

The special feature of this method is the ability to automatically start on the target system a linux live OS and at the end of the copy process to perform the necessary changes to make bootable the new system. Once booted the rebuilt machine on VM, this will be ready to be replaced to the original one. It 'also duty of the client to automatically switch between the two machines (the new one on VM and the old one on physical platform) avoiding to create conflicts of IP address on the LAN. 


\section{Use cases}

In the distributed computing laboratory (LCD) at IAPS-INAF, we applied the methodology described above on two different contexts explained in detail in the following.

\subsection{AVES master controllers}

AVES is the computer cluster system adopted to perform the analisys of data provided by INTEGRAL scientific satellite. The most critical point of failure of that system is focused on the behavior of master controllers devoted to the computing nodes control (see Figure 1). The problem was been solved by the creation of two different VMs in HA mode running the clone images of the two master controller. The two VMs are hosted on a XEN-SERVER 6.2 2.

\subsection{Data Storage Subsystem}

INTEGRAL Data Storage Subsystem (DSS) [4] performs the following duties:

- downloads data from IDSC

- backups the data in real time

- provides the data to AVES

- In case of fault:

- data download stops

- the data backup is no longer synchronized

- every running scientific data analysis is aborted

The DSS is hosted by two VMs in HA mode to ensure the availability of data (16TB) and their synchronization with the central server (ISDC) to prevent the problems explained above.

\section{Conclusions}

This system through the creation of virtual machines from physical machines in active service, allows to increase very much their reliability. The ease and speed with which VMs are made active greatly improves maintenance time systems hosted. The low cost of the individual VM makes it extremely interesting for the implementation of host virtualized systems of many servers that make up the facility of calculation of small and medium sized structures. The portability of VMs between different virtualization platforms makes the unavoidable transition to new generation architectures (because of the well known and continuous technological improvements) easy and economic from the management point of view.

Acknowledgments Pietro Ubertini IAPS Director Franco Giovannelli and staff 


\section{References}

[1] Winkler, C., et al., The INTEGRAL mission. Astron. Astrophys. 411, L1L6 (2003)

[2] Federici, M., Gaibisso, C., Martino, B. L., "HAVmS: Highly Available Virtual Machine Computer System Fault Tolerant with Automatic Failback and Close to Zero Downtime", Proceedings of the The Tenth International Workshop on Multifrequency Behaviour of High Energy Cosmic Sources, Palermo, Italy, 2013, p.278-282

[3] Federici, M., Martino, B. L., Ubertini, P., "AVES: A high performance computer cluster array for the INTEGRAL satellite scientific data analysis", Experimental Astronomy Volume 34, p. 105-121

[4] Martino, B. L., Federici, M., "An high availability data storage subsystem for the INTEGRAL data analysis", Mem. S.A.It. Vol. 75, 282 (2008) 